\title{
Transverse momentum dependent splitting functions at work: quark-to-gluon splitting
}

\author{
M. Hentschinski, ${ }^{1,2}$ A. Kusina, ${ }^{3}$ and K. $\operatorname{Kutak}^{4}$ \\ ${ }^{1}$ Facultad de Ciencias Físico Matemáticas, Benemérita Universidad Autónoma de Puebla, \\ Puebla 1152, Mexico \\ ${ }^{2}$ Instituto de Ciencias Nucleares, Universidad Nacional Autónoma de México, \\ Apartado Postal 70-543, Cuidad de México 04510, Mexico \\ ${ }^{3}$ Laboratoire de Physique Subatomique et de Cosmologie \\ 53 Rue des Martyrs Grenoble, France \\ ${ }^{4}$ Instytut Fizyki Jadrowej im. H. Niewodniczańskiego, \\ Radzikowskiego 152, 31-342 Kraków, Poland
}

\begin{abstract}
Using the recently obtained $P_{g q}$ splitting function we extend the low $x$ evolution equation for gluons to account for contributions originating from quark-to-gluon splitting. In order to write down a consistent equation we resum virtual corrections coming from the gluon channel and demonstrate that this implies a suitable regularization of the $P_{g q}$ singularity, corresponding to a soft emitted quark. We also note that the obtained equation is in a straightforward manner generalized to a nonlinear evolution equation which takes into account effects due to the presence of high gluon densities.
\end{abstract}

\section{CONTENTS}

\section{Introduction}

II. The TMD quark-to-gluon splitting function

III. Combining quarks and gluons
A. Gluon part
B. Quark part
C. Combined gluon and quark parts
D. Numerical studies
E. Low $x$ limit

IV. Summary and outlook

Acknowledgments

References

\section{INTRODUCTION}

Parton distribution functions (PDFs) provide essential input to phenomenology at today's collider experiments. In combination with partonic cross-sections, which can be systematically calculated within QCD perturbation theory, they allow for a very accurate descriptions of 'hard' events in hadron-hadron and hadron-electron collisions, where 'hard' refers to the presence of a scale $M$ significantly larger than typical hadronic scales of the order of $\Lambda_{\mathrm{QCD}} \sim 200 \mathrm{MeV}$. While the bulk of such analysis is carried out within the framework of collinear factorization, there exist classes of multi-scale processes where the use of more general schemes is of advantage. Such schemes involve in general Transverse-MomentumDependent (TMD) $)^{1}$ or 'unintegrated' PDFs in contrast

\footnotetext{
${ }^{1}$ For a review see 1 .
}

to conventional PDFs defined within collinear factorization which depend only on the hadron longitudinal momentum fraction carried by the parton.

A particularly interesting example of such a multiscale process is provided by the high-energy or low $x$ limit of hard processes $s \gg M^{2} \gg \Lambda_{\mathrm{QCD}}^{2}$ where $\sqrt{s}$ denotes the center-of-mass energy of the process and $x=M^{2} / s$. In such a scenario it is necessary to resum terms enhanced by logarithms $\ln 1 / x$ to all orders in the strong coupling constant $\alpha_{s}$, which is achieved by the Balitsky-Fadin-Kuraev-Lipatov (BFKL) [2, 3] evolution equation. The resulting formalism called high energy factorization or $k_{T}$ factorization [4-7] provides then a factorization of such cross-sections into a TMD coefficient 8 8-10, or 'impact factor' and an 'unintegrated' gluon density.

While well defined in the 'low $x$ ' limit, the ensuing formalism and evolution equation of the unintegrated gluon density is confronted with difficulties if one attempts a naïve extension into the 'large or moderate $x$ ' region. In particular, this concerns implementations of unintegrated parton densities in parton showers of Monte-Carlo event generators, as well as observables in hadron-hadron collisions and/or combinations with fragmentation functions which involve integrals over the full $x$ range of initial state PDFs. One of the most tantalizing deficits is the limitation to gluon-to-gluon splittings in the low $x$ evolution with quarks being absent. While well justified in the limit $x \ll 1$, this restriction omits a resummation of collinear logarithms associated with quark splittings which provide sizable contributions at intermediate and large $x$. Furthermore, some of the hard collision processes are initiated by quarks [11] and therefore the appropriate unintegrated parton density functions are needed [12]. To overcome this limitation, in [13] the real parts of quark induced TMD splitting functions have been calculated within $k_{T}$-factorization, supplementing earlier results 
of Catani and Hautmann [14, 15, who calculated the TMD gluon-to-quark splitting function. This calculation has been based on an extension of the method of the classical work by Curci Furmanski and Petronzio [16, formulated for the collinear factorization ${ }^{2}$ Together with the already long-known $k_{T}$-dependent $P_{g g}$ splitting function [20 23] it gives a complete set of (real) emission evolution kernels within the $k_{T}$-factorization scheme.

One of the peculiarities of the quark-induced splitting kernels is that they develop a singularity, which is associated with the vanishing of the transverse momentum of the parton emitted during the splitting. While in the case of the quark-to-quark splitting the resulting singularity is expected to be cancelled by the corresponding virtual corrections, the situation is less clear in the case of the quark-to-gluon splitting, where such virtual correction is absent at leading order. While a complete treatment of this singularity requires eventually the development of a suitable scheme which removes this singularity, we will address in the following a different question. Namely, whether it is possible to formulate evolution equations which, due to their particular structure, regularize this divergence.

To this end we formulate an evolution equation for the gluon distribution which includes already the quarkto-gluon splitting and therefore first corrections due to quarks. Besides of testing possible implementation of the quark-to-gluon splitting function, this equation will allow us - even before the complete set of equations is known - to investigate the impact of including quarks into the purely gluonic picture given by BFKL like evolution equations.

We should also note that one of the possibilities to construct a set of TMD parton distributions is to use the Kimber Martin Ryskin framework [24, 25]. In this approach one starts from collinear PDFs and constructs the TMD PDFs by introducing coherence effects in the last step of the evolution. The widely used PDF sets constructed in this manner are used rather sucessfully in phenomenological studies [26-30. However, the potential problem which one faces is the limitation to rather large and moderate values of $x$ and also large transversal momenta. Therefore, one can not address questions related to gluon saturation and the impact of gluon saturation on quarks.

The rest of the paper is organized as follows. In Sec. III we remind the result for the $P_{g q}$ splitting function obtained in 13 and adopt it to a form suitable for the current work. In Sec. III we formulate a generalization of

\footnotetext{
2 The Curci Furmanski Petronzio scheme was recently modified in order to simplify the structure of infra-red singularities, and used for calculating inclusive as well as unintegrated NLO splitting functions for the purpose of MC simulations (these results are in the framework of collinear factorization) [17-19.
}

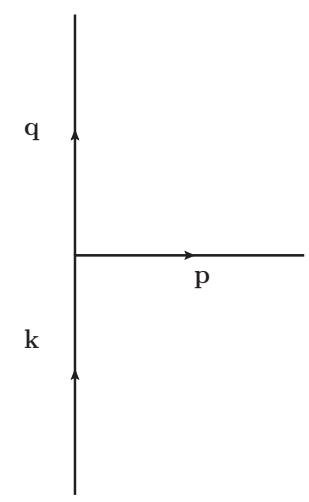

FIG. 1. An initial state parton with transverse momentum $\mathbf{k}$ splits into a parton with transverse momentum $\mathbf{q}$, while emitting a real parton with momentum $\mathbf{p}=\mathbf{k}-\mathbf{q}$.

BFKL equation including quark contributions from the $P_{g q}$ splitting and perform partial resummation of this contribution. Next the numerical stability of this new equation is studied. Finally we calculate its high energy limit and show that in this limit we obtain a full resummation of the quark part. In Sec. [IV] we summarize the obtained results and give perspective for further studies.

\section{THE TMD QUARK-TO-GLUON SPLITTING FUNCTION}

We start by recalling the results of $[13$, where the TMD quark-to-gluon splitting function $P_{g q}$ has been derived, using an extension of the $k_{T}$-factorization formalism to initial, off-shell quarks. The corresponding kinematics is depicted in Fig. 1. The result of [13] is at first provided in terms of the so-called TMD splitting kernel which allow to identify the splitting functions of interest. Since we are in the following in particular interested in isolating the singularity associated with the vanishing transverse momentum $\mathbf{p}$ of the real emitted quark we start with a representation in terms of the rescaled transverse momentum $\tilde{\mathbf{p}}=\frac{\mathbf{k}-\mathbf{q}}{1-z}$. The TMD splitting kernel is then given by

$$
\begin{gathered}
\hat{K}_{g q}\left(z, \frac{\mathbf{k}^{2}}{\mu_{F}^{2}}, \alpha_{s}, \epsilon\right)= \\
\frac{\alpha_{s}}{2 \pi} z \int \frac{d^{2+2 \epsilon} \tilde{\mathbf{p}}}{\pi^{1+\epsilon} \mu^{2 \epsilon}} e^{-\epsilon \gamma_{E}} \Theta\left(\mu_{F}^{2}-(1-z)(\tilde{\mathbf{p}}-\mathbf{k})^{2}-z \mathbf{k}^{2}\right) \\
\left\{\frac{(1-z)^{2 \epsilon}}{z} \frac{2}{\tilde{\mathbf{p}}^{2}}+\frac{\tilde{\mathbf{p}}^{2} z(1-z)^{2+2 \epsilon}(1+\epsilon)}{\left[(1-z)(\tilde{\mathbf{p}}-\mathbf{k})^{2}+z \mathbf{k}^{2}\right]^{2}}\right. \\
\left.\quad-\frac{2(1-z)^{1+2 \epsilon}}{(1-z)(\tilde{\mathbf{p}}-\mathbf{k})^{2}+z \mathbf{k}^{2}}\right\} .
\end{gathered}
$$

For the purpose of the following sections, we rewrite this result using the transverse momenta of the emitted particle, $\mathbf{p}$, and the outgoing particle, $\mathbf{q}$, while $\mathbf{k}=\mathbf{p}+\mathbf{q}$. 
Furthermore, since we will formulate the evolution equation in $d=4$ dimensions, we set from now on $\epsilon=0$. We find

$$
\begin{aligned}
& \hat{K}_{g q}\left(z, \frac{\mathbf{q}^{2}}{\mu_{F}^{2}}, \alpha_{s}, 0\right)= \\
& \frac{\alpha_{s}}{2 \pi} z \int \frac{d^{2} \mathbf{p}}{\pi} \Theta\left(\mu_{F}^{2}-\frac{z \mathbf{p}^{2}+(1-z) \mathbf{q}^{2}}{1-z}\right) \frac{P_{g q}(z, \mathbf{p}, \mathbf{q})}{\mathbf{p}^{2}}
\end{aligned}
$$

where

$$
P_{g q}(z, \mathbf{p}, \mathbf{q})=C_{F} \frac{\mathbf{p}^{4} z^{2}-2 \mathbf{p}^{2} \mathbf{q}^{2}(z-1) z+2 \mathbf{q}^{4}(1-z)^{2}}{z\left(\mathbf{q}^{2}(1-z)+\mathbf{p}^{2} z\right)^{2}} .
$$

In the limit $\mathbf{p} \rightarrow 0$, the splitting function reduces to

$$
\lim _{\mathbf{p} \rightarrow 0} P_{g q}(z, \mathbf{p}, \mathbf{q})=\frac{2 C_{F}}{z}
$$

and as a consequence, Eq. 2.4 appears to develop a singularity in this limit. We stress that this singularity is not to be confused with the conventional collinear singularity, which is associated with the collinear gluonto-quark splitting function. As demonstrated in [13], the collinear singularity is associated with the collinear limit $|\mathbf{p}|,|\mathbf{q}| \gg|\mathbf{k}|$. In the following section we will present a possible implementation of this splitting function into an evolution equation for the unintegrated gluon density, taking particular care to avoid the dangerous region $|\mathbf{p}| \rightarrow 0$.

\section{COMBINING QUARKS AND GLUONS}

Our starting point is the leading order (LO) BFKL equation which describes evolution in $\ln 1 / x$ for the dipole amplitude in the momentum space:

$$
\begin{aligned}
& \mathcal{F}(x,\left.\mathbf{q}^{2}\right)=\mathcal{F}^{0}\left(x, \mathbf{q}^{2}\right)+\bar{\alpha}_{s} \int_{x}^{1} \frac{d z}{z} \int \frac{d^{2} \mathbf{p}}{\pi \mathbf{p}^{2}} \\
& \times\left[\mathcal{F}\left(x / z,|\mathbf{q}+\mathbf{p}|^{2}\right)-\theta\left(\mathbf{q}^{2}-\mathbf{p}^{2}\right) \mathcal{F}\left(x / z, \mathbf{q}^{2}\right)\right]
\end{aligned}
$$

where $\bar{\alpha}_{s}=\frac{C_{A} \alpha_{s}}{\pi}$. This form is particularly useful to promote the BFKL equation to a system of equations for quarks and gluons. First of all let us consider the equation for gluons at LO which apart from gluons receives also contributions from quarks. Formally such an equation can be formulated as:

$$
\begin{aligned}
\mathcal{F}\left(x, \mathbf{q}^{2}\right) & =\mathcal{F}^{0}\left(x, \mathbf{q}^{2}\right)+\bar{\alpha}_{s} P_{B F K L}(z, \mathbf{p}, \mathbf{q}) \otimes \mathcal{F}\left(x / z, \mathbf{q}^{2}\right) \\
& +\frac{\alpha_{s}}{2 \pi} P_{g q}(z, \mathbf{p}, \mathbf{q}) \otimes \mathcal{Q}\left(x / z, \mathbf{q}^{2}\right)
\end{aligned}
$$

where $\mathcal{Q}\left(x / z, \mathbf{q}^{2}\right)$ is the distribution of quarks and $P_{g q}$ is given by eq. (2.4). Introducing the resolution scale $\mu$ and decomposing the kernel of the gluonic part of (3.1) into a resolved real emission part with $\mathbf{p}^{2}>\mu^{2}$ and the unresolved part with $\mathbf{p}^{2}<\mu^{2}$, we obtain

$$
\begin{aligned}
\mathcal{F}\left(x, \mathbf{q}^{2}\right) & =\mathcal{F}^{0}\left(x, \mathbf{q}^{2}\right) \\
& +\bar{\alpha}_{s} \int_{x}^{1} \frac{d z}{z} \int \frac{d^{2} \mathbf{p}}{\pi \mathbf{p}^{2}} \mathcal{F}\left(x / z,|\mathbf{q}+\mathbf{p}|^{2}\right) \theta\left(\mathbf{p}^{2}-\mu^{2}\right) \\
& +\bar{\alpha}_{s} \int_{x}^{1} \frac{d z}{z} \int \frac{d^{2} \mathbf{p}}{\pi \mathbf{p}^{2}}\left[\mathcal{F}\left(x / z,|\mathbf{q}+\mathbf{p}|^{2}\right) \theta\left(\mu^{2}-\mathbf{p}^{2}\right)\right. \\
& \left.-\theta\left(\mathbf{q}^{2}-\mathbf{p}^{2}\right) \mathcal{F}\left(x / z, \mathbf{q}^{2}\right)\right] \\
& +\frac{\alpha_{s}}{2 \pi} \int_{x}^{1} d z \int \frac{d^{2} \mathbf{p}}{\pi \mathbf{p}^{2}} P_{g q}(z, \mathbf{p}, \mathbf{q}) \mathcal{Q}\left(x / z,|\mathbf{p}+\mathbf{q}|^{2}\right)
\end{aligned}
$$

The integral over $\mathbf{p}$ in the quark part is divergent and needs to be regulated. In the following we achieve this through introducing the same cut-off $\mu$ as used for the gluonic part. Technically this is achieved through including for the quark part a theta-function $\theta\left(\mathbf{p}^{2}-\mu^{2}\right) 3^{3}$

A convenient way of formulating the resummation (or exponentiation) of virtual and unresolved real emissions in the case of the gluon part can be achieved by expressing the evolution equation by its Mellin transform which is then further manipulated [31]. The Mellin transform is defined as

$$
\begin{aligned}
& \overline{\mathcal{F}}\left(\omega, \mathbf{q}^{2}\right)=\int_{0}^{1} d x x^{\omega-1} \mathcal{F}\left(x, \mathbf{q}^{2}\right), \\
& \overline{\mathcal{Q}}\left(\omega, \mathbf{q}^{2}\right)=\int_{0}^{1} d x x^{\omega-1} \mathcal{Q}\left(x, \mathbf{q}^{2}\right)
\end{aligned}
$$

while the inverse transform reads

$$
\begin{aligned}
& \mathcal{F}\left(x, \mathbf{q}^{2}\right)=\frac{1}{2 \pi i} \int_{c-i \infty}^{c+i \infty} d \omega x^{-\omega} \overline{\mathcal{F}}\left(\omega, \mathbf{q}^{2}\right), \\
& \mathcal{Q}\left(x, \mathbf{q}^{2}\right)=\frac{1}{2 \pi i} \int_{c-i \infty}^{c+i \infty} d \omega x^{-\omega} \overline{\mathcal{Q}}\left(\omega, \mathbf{q}^{2}\right) .
\end{aligned}
$$

Performing the Mellin transform and using in the unresolved part $|\mathbf{q}+\mathbf{p}|^{2} \approx \mathbf{q}^{2}\left(\right.$ since $\left.\mathbf{p}^{2}<\mu^{2}\right)$ we obtain

$$
\begin{aligned}
\overline{\mathcal{F}}\left(\omega, \mathbf{q}^{2}\right) & =\overline{\mathcal{F}}^{0}\left(\omega, \mathbf{q}^{2}\right) \\
& +\frac{\bar{\alpha}_{s}}{\omega} \int \frac{d^{2} \mathbf{p}}{\pi \mathbf{p}^{2}}\left[\overline{\mathcal{F}}\left(\omega,|\mathbf{q}+\mathbf{p}|^{2}\right) \theta\left(\mathbf{p}^{2}-\mu^{2}\right)\right] \\
& +\frac{\bar{\alpha}_{s}}{\omega} \int \frac{d^{2} \mathbf{p}}{\pi \mathbf{p}^{2}} \overline{\mathcal{F}}\left(\omega, \mathbf{q}^{2}\right)\left[\theta\left(\mu^{2}-\mathbf{p}^{2}\right)-\theta\left(\mathbf{q}^{2}-\mathbf{p}^{2}\right)\right] \\
& +\frac{\alpha_{s}}{2 \pi} \int \frac{d^{2} \mathbf{p}}{\pi \mathbf{p}^{2}} \bar{P}_{g q}(\omega, \mathbf{q}, \mathbf{p}) \overline{\mathcal{Q}}\left(\omega,|\mathbf{q}+\mathbf{p}|^{2}\right) \theta\left(\mathbf{p}^{2}-\mu^{2}\right)
\end{aligned}
$$

where

$$
\bar{P}_{g q}(\omega, \mathbf{p}, \mathbf{q})=\int_{0}^{1} d z P_{g q}(z, \mathbf{p}, \mathbf{q}) z^{\omega}
$$

For the moment the Mellin transform of the quark part is treated as a formal expressions giving a convenient shorthand notation. After combining the unresolved real and virtual parts of the gluonic terms we obtain 


$$
\begin{aligned}
\overline{\mathcal{F}}\left(\omega, \mathbf{q}^{2}\right) & =\overline{\mathcal{F}}^{0}\left(\omega, \mathbf{q}^{2}\right) \\
& +\frac{\bar{\alpha}_{s}}{\omega} \int \frac{d^{2} \mathbf{p}}{\pi \mathbf{p}^{2}} \overline{\mathcal{F}}\left(\omega,|\mathbf{q}+\mathbf{p}|^{2}\right) \theta\left(\mathbf{p}^{2}-\mu^{2}\right)-\frac{\bar{\alpha}_{s}}{\omega} \overline{\mathcal{F}}\left(\omega, \mathbf{q}^{2}\right) \ln \frac{\mathbf{q}^{2}}{\mu^{2}} \\
& +\frac{\alpha_{s}}{2 \pi} \int \frac{d^{2} \mathbf{p}}{\pi \mathbf{p}^{2}} P_{g q}(\omega, \mathbf{p}, \mathbf{q}) \overline{\mathcal{Q}}\left(\omega,|\mathbf{q}+\mathbf{p}|^{2}\right) \theta\left(\mathbf{p}^{2}-\mu^{2}\right) .
\end{aligned}
$$

With

$$
\hat{\mathcal{F}}^{0}\left(\omega, \mathbf{q}^{2}\right)=\frac{\omega}{\omega+\bar{\omega}} \overline{\mathcal{F}}^{0}\left(\omega, \mathbf{q}^{2}\right), \quad \bar{\omega}=\bar{\alpha}_{s} \ln \frac{\mathbf{q}^{2}}{\mu^{2}} .
$$

This can be simplified to

$$
\begin{aligned}
\overline{\mathcal{F}}\left(\omega, \mathbf{q}^{2}\right) & =\hat{\mathcal{F}}^{0}\left(\omega, \mathbf{q}^{2}\right) \\
& +\frac{\bar{\alpha}_{s}}{\bar{\omega}+\omega} \int \frac{d^{2} \mathbf{p}}{\pi \mathbf{p}^{2}} \overline{\mathcal{F}}\left(\omega,|\mathbf{q}+\mathbf{p}|^{2}\right) \theta\left(\mathbf{p}^{2}-\mu^{2}\right) \\
& +\frac{\alpha_{s}}{2 \pi} \frac{\omega}{\omega+\bar{\omega}} \int \frac{d^{2} \mathbf{p}}{\pi \mathbf{p}^{2}} P_{g q}(\omega, \mathbf{p}, \mathbf{q}) \overline{\mathcal{Q}}\left(\omega,|\mathbf{q}+\mathbf{p}|^{2}\right) \theta\left(\mathbf{p}^{2}-\mu^{2}\right)
\end{aligned}
$$

which is easily verified through multiplying eq. 3.10 by a factor $\frac{\bar{\omega}+\omega}{\omega}$.

Now we will transform this expression back to $x$ space. We will do this separately for the gluon and quark parts. We start by writing the formal expression

$$
\begin{aligned}
\mathcal{F}\left(x, \mathbf{q}^{2}\right) & =\frac{1}{2 \pi i} \int_{c-i \infty}^{c+i \infty} d \omega x^{-\omega} \hat{\mathcal{F}}^{0}\left(\omega, \mathbf{q}^{2}\right) \\
+ & \left.\frac{1}{2 \pi i} \int_{c-i \infty}^{c+i \infty} d \omega x^{-\omega} \frac{\bar{\alpha}_{s}}{\omega+\bar{\omega}} \int \frac{d^{2} \mathbf{p}}{\pi \mathbf{p}^{2}} \int_{0}^{1} d y y^{\omega-1} \mathcal{F}\left(y,|\mathbf{q}+\mathbf{p}|^{2}\right)\right] \theta\left(\mathbf{p}^{2}-\mu^{2}\right) \\
+ & \frac{1}{2 \pi i} \int_{c-i \infty}^{c+i \infty} d \omega x^{-\omega} \frac{\alpha_{s}}{2 \pi} \frac{\omega}{\omega+\bar{\omega}} \int \frac{d^{2} \mathbf{p}}{\pi \mathbf{p}^{2}} \int_{0}^{1} d z z^{\omega} P_{g q}(z, \mathbf{p}, \mathbf{q}) \\
& \times \int_{0}^{1} d y y^{\omega-1} \mathcal{Q}\left(y,|\mathbf{q}+\mathbf{p}|^{2}\right) \theta\left(\mathbf{p}^{2}-\mu^{2}\right)
\end{aligned}
$$

\section{A. Gluon part}

For the part with the initial gluon distribution, eq. (3.11), we merely define

$$
\tilde{\mathcal{F}}^{0}\left(x, \mathbf{q}^{2}\right) \equiv \frac{1}{2 \pi i} \int_{c-i \infty}^{c+i \infty} d \omega x^{-\omega} \hat{\mathcal{F}}^{0}\left(\omega, \mathbf{q}^{2}\right) .
$$

To transform the remaining gluonic part given by eq. 3.12 it is convenient to first note that

$$
\frac{1}{\omega+\bar{\omega}}=\int_{0}^{1} \frac{d z}{z} z^{\omega+\bar{\omega}}
$$

Using this identity we find that the gluonic term transforms into

$$
\begin{aligned}
& \left.\frac{1}{2 \pi i} \int_{c-i \infty}^{c+i \infty} d \omega x^{-\omega} \bar{\alpha}_{s} \int_{0}^{1} \frac{d z}{z} z^{\omega+\bar{\omega}} \int \frac{d^{2} \mathbf{p}}{\pi \mathbf{p}^{2}} \int_{0}^{1} d y y^{\omega-1} \mathcal{F}\left(y,|\mathbf{q}+\mathbf{p}|^{2}\right)\right] \theta\left(\mathbf{p}^{2}-\mu^{2}\right) \\
& =\bar{\alpha}_{s} \int \frac{d^{2} \mathbf{p}}{\pi \mathbf{p}^{2}} \int_{0}^{1} \frac{d z}{z} z^{\bar{\omega}} \int_{0}^{1} \frac{d y}{y} \mathcal{F}\left(y,|\mathbf{q}+\mathbf{p}|^{2}\right) \theta\left(\mathbf{p}^{2}-\mu^{2}\right) \delta\left(\frac{z y}{x}-1\right) \\
& =\bar{\alpha}_{s} \int \frac{d^{2} \mathbf{p}}{\pi \mathbf{p}^{2}} \int_{x}^{1} \frac{d z}{z} \Delta_{R}\left(z, \mathbf{q}^{2}, \mu^{2}\right) \mathcal{F}\left(\frac{x}{z},|\mathbf{q}+\mathbf{p}|^{2}\right) \theta\left(\mathbf{p}^{2}-\mu^{2}\right)
\end{aligned}
$$

\footnotetext{
${ }^{3}$ Whereas, in the gluon case, this additional scale is just for technical convenience as $1 / \mathbf{p}^{2}$ is regularized by the virtual contribution,
}

in the case of quarks, $\mu$ scale is really needed for regularizing the corresponding expression. 
where we have introduced the Regge formfactor

$$
\Delta_{R}\left(z, \mathbf{q}^{2}, \mu^{2}\right) \equiv \exp \left(-\bar{\alpha}_{s} \ln \frac{1}{z} \ln \frac{\mathbf{q}^{2}}{\mu^{2}}\right)
$$

which ensures that the gluonic part is well-behaving when $\mu^{2} \rightarrow 0$ and therefore allows us to take $\mathbf{p}^{2} \rightarrow 0$.

\section{B. Quark part}

Now we turn to the quark part given by formula 3.13 . We note that for a generic function $f(\omega)$ we have

$$
\int \frac{d \omega}{2 \pi i} \omega x^{-\omega} f(\omega)=-x \partial_{x} \int \frac{d \omega}{2 \pi i} x^{-\omega} f(\omega) .
$$

We additionally isolate the part singular in $z$ of the $P_{g q}$ kernel

$$
P_{g q}(z, \mathbf{p}, \mathbf{q}) \equiv \frac{1}{z} \cdot \tilde{P}_{g q}(z, \mathbf{p}, \mathbf{q})
$$

This allows us to rewrite eq. (3.13) as

$$
\begin{aligned}
& -x \partial_{x} \frac{\alpha_{s}}{2 \pi} \int \frac{d \omega}{2 \pi i} x^{-\omega} \int_{0}^{1} \frac{d z_{1}}{z_{1}} z_{1}^{\omega+\bar{\omega}} \int \frac{d^{2} \mathbf{p}}{\pi \mathbf{p}^{2}} \int_{0}^{1} \frac{d z_{2}}{z_{2}} z_{2}^{\omega} \tilde{P}_{g q}\left(z_{2}, \mathbf{p}, \mathbf{q}\right) \\
& =-x \partial_{x} \frac{\alpha_{s}}{2 \pi} \int \frac{d^{2} \mathbf{p}}{\pi \mathbf{p}^{2}} \int_{0}^{1} \frac{d z_{1}}{z_{1}} z_{1}^{\bar{\omega}} \int_{0}^{1} \frac{d z_{2}}{z_{2}} \tilde{P}_{g q}\left(z_{2}, \mathbf{p}, \mathbf{q}\right) \\
& =-x \partial_{x} \frac{\alpha_{s}}{2 \pi} \int \frac{d^{2} \mathbf{p}}{\pi \mathbf{p}^{2}} \int_{0}^{1} \frac{d z_{1}}{z_{1}} z_{1}^{\bar{\omega}} \int_{0}^{1} \frac{d y}{y} \theta\left(y,|\mathbf{q}+\mathbf{p}|^{2}\right) \theta\left(\mathbf{p}^{2}-\mu^{2}\right) \\
& =
\end{aligned}
$$

Next note that

$$
\begin{aligned}
-x \partial_{x} \theta\left(y z_{1}-x\right) & \tilde{P}_{g q}\left(\frac{x}{y z_{1}}, \mathbf{p}, \mathbf{q}\right) \\
& =x \delta\left(x-y z_{1}\right) \tilde{P}_{g q}(1, \mathbf{p}, \mathbf{q})-\theta\left(y z_{1}-x\right)\left[\tilde{P}_{g q}^{\prime}\left(\frac{x}{y z_{1}}, \mathbf{p}, \mathbf{q}\right) \frac{x}{y z_{1}}\right]
\end{aligned}
$$

where

$$
\tilde{P}_{g q}^{\prime}(z, \mathbf{p}, \mathbf{q}) \equiv \frac{d}{d z} \tilde{P}_{g q}(z, \mathbf{p}, \mathbf{q})=-C_{F} \frac{2(1-z) \mathbf{p}^{2} \mathbf{q}^{4}}{\left(z \mathbf{p}^{2}+(1-z) \mathbf{q}^{2}\right)^{3}}
$$

Now using eq. 3.22 we obtain for the quark contribution

$$
\begin{aligned}
& \frac{\alpha_{s}}{2 \pi} \int \frac{d^{2} \mathbf{p}}{\pi \mathbf{p}^{2}} \int_{x}^{1} \frac{d z}{z} z^{\bar{\omega}} \tilde{P}_{g q}(1, \mathbf{p}, \mathbf{q}) \mathcal{Q}\left(\frac{x}{z},|\mathbf{q}+\mathbf{p}|^{2}\right) \theta\left(\mathbf{p}^{2}-\mu^{2}\right) \\
& -\frac{\alpha_{s}}{2 \pi} \int \frac{d^{2} \mathbf{p}}{\pi \mathbf{p}^{2}} \int_{x}^{1} \frac{d z}{z} \int_{z}^{1} \frac{d z_{1}}{z_{1}} z_{1}^{\bar{\omega}}\left[\tilde{P}_{g q}^{\prime}\left(\frac{z}{z_{1}}, \mathbf{p}, \mathbf{q}\right) \frac{z}{z_{1}}\right] \mathcal{Q}\left(\frac{x}{z},|\mathbf{q}+\mathbf{p}|^{2}\right) \theta\left(\mathbf{p}^{2}-\mu^{2}\right)
\end{aligned}
$$

Note that $\tilde{P}_{g q}(1, \mathbf{p}, \mathbf{q})=C_{F}$. While the convolution integral over $z$ involves the (non-perturbative) unintegrated quark density, the $z_{1}$ integral (in the second line) can in principle be calculated analytically. 


\section{Combined gluon and quark parts}

Combining the results for the gluon part (3.17) and quark part 3.24 we obtain

$$
\begin{aligned}
\mathcal{F}\left(x, \mathbf{q}^{2}\right) & =\tilde{\mathcal{F}}^{0}\left(x, \mathbf{q}^{2}\right) \\
& +\bar{\alpha}_{s} \int \frac{d^{2} \mathbf{p}}{\pi \mathbf{p}^{2}} \int_{x}^{1} \frac{d z}{z} \Delta_{R}\left(z, \mathbf{q}^{2}, \mu^{2}\right) \mathcal{F}\left(\frac{x}{z},|\mathbf{q}+\mathbf{p}|^{2}\right) \theta\left(\mathbf{p}^{2}-\mu^{2}\right) \\
& +\frac{\alpha_{s}}{2 \pi} \int \frac{d^{2} \mathbf{p}}{\pi \mathbf{p}^{2}} \int_{x}^{1} \frac{d z}{z} z^{\bar{\omega}} \tilde{P}_{g q}(1, \mathbf{p}, \mathbf{q}) \mathcal{Q}\left(\frac{x}{z},|\mathbf{q}+\mathbf{p}|^{2}\right) \theta\left(\mathbf{p}^{2}-\mu^{2}\right) \\
& -\frac{\alpha_{s}}{2 \pi} \int \frac{d^{2} \mathbf{p}}{\pi \mathbf{p}^{2}} \int_{x}^{1} \frac{d z}{z} \int_{z}^{1} \frac{d z_{1}}{z_{1}} z_{1}^{\bar{\omega}}\left[\tilde{P}_{g q}^{\prime}\left(\frac{z}{z_{1}}, \mathbf{p}, \mathbf{q}\right) \frac{z}{z_{1}}\right] \mathcal{Q}\left(\frac{x}{z},|\mathbf{q}+\mathbf{p}|^{2}\right) \theta\left(\mathbf{p}^{2}-\mu^{2}\right) .
\end{aligned}
$$

Note that $z^{\bar{\omega}}=\Delta_{R}\left(z, \mathbf{q}^{2}, \mu^{2}\right)$. Using in addition $\tilde{P}_{g q}(1, \mathbf{p}, \mathbf{q})=C_{F}$, we obtain

$$
\begin{aligned}
& \mathcal{F}\left(x, \mathbf{q}^{2}\right)= \tilde{\mathcal{F}}^{0}\left(x, \mathbf{q}^{2}\right) \\
&+\frac{\alpha_{s}}{2 \pi} \int_{x}^{1} \frac{d z}{z} \int \frac{d^{2} \mathbf{p}}{\pi \mathbf{p}^{2}} \theta\left(\mathbf{p}^{2}-\mu^{2}\right) {[} \\
& \Delta_{R}\left(z, \mathbf{q}^{2}, \mu^{2}\right)\left(2 C_{A} \mathcal{F}\left(\frac{x}{z},|\mathbf{q}+\mathbf{p}|^{2}\right)+C_{F} \mathcal{Q}\left(\frac{x}{z},|\mathbf{q}+\mathbf{p}|^{2}\right)\right) \\
&\left.-\int_{z}^{1} \frac{d z_{1}}{z_{1}} \Delta_{R}\left(z_{1}, \mathbf{q}^{2}, \mu^{2}\right)\left[\tilde{P}_{g q}^{\prime}\left(\frac{z}{z_{1}}, \mathbf{p}, \mathbf{q}\right) \frac{z}{z_{1}}\right] \mathcal{Q}\left(\frac{x}{z},|\mathbf{q}+\mathbf{p}|^{2}\right)\right]
\end{aligned}
$$

From the above expression we can see that the $\frac{1}{\mathbf{p}^{2}}$ singularity of the quark term in the third line of eq. 3.26) is regularized by the Regge formfactor in direct analogy with the gluonic term. For the term in the fourth line we note on the other hand that $\tilde{P}_{g q}^{\prime}(z, \mathbf{p}, \mathbf{q}) \sim \mathbf{p}^{2}$ for finite $\mathbf{q}$; the limit $\mu^{2} \rightarrow 0$ is therefore expected to be finite for this term. Before addressing the numerical stability in the next section, we note that the above resummation can be in a straight forward manner extended [32, 33] to the situation where the gluon density is large and therefore subject to a nonlinear evolution equation, taking into account saturation effects [34, 35. The extended non-linear equation reads:

$$
\begin{aligned}
& \mathcal{F}\left(x, \mathbf{q}^{2}\right)=\tilde{\mathcal{F}}^{0}\left(x, \mathbf{q}^{2}\right)+\frac{\alpha_{s}}{2 \pi} \int_{x}^{1} \frac{d z}{z} \int \frac{d^{2} \mathbf{p}}{\pi \mathbf{p}^{2}} \theta\left(\mathbf{p}^{2}-\mu^{2}\right) \\
& \Delta_{R}\left(z, \mathbf{q}^{2}, \mu^{2}\right)\left\{2 C_{A} \mathcal{F}\left(\frac{x}{z},|\mathbf{q}+\mathbf{p}|^{2}\right)+C_{F} \mathcal{Q}\left(\frac{x}{z},|\mathbf{q}+\mathbf{p}|^{2}\right)\right. \\
&\left.-\frac{4 \pi \alpha_{s}}{R^{2}} \mathbf{p}^{2} \delta\left(\mathbf{p}^{2}-\mathbf{q}^{2}\right)\left[\left(\int_{\mathbf{p}^{2}}^{\infty} \frac{\mathrm{d} l^{2}}{l^{2}} \mathcal{F}\left(\frac{x}{z}, l^{2}\right)\right)^{2}+\mathcal{F}\left(\frac{x}{z}, \mathbf{p}^{2}\right) \int_{\mathbf{p}^{2}}^{\infty} \frac{\mathrm{d} l^{2}}{l^{2}} \ln \left(\frac{l^{2}}{\mathbf{p}^{2}}\right) \mathcal{F}\left(\frac{x}{z}, l^{2}\right)\right]\right\} \\
&\left.-\int_{z}^{1} \frac{d z_{1}}{z_{1}} \Delta_{R}\left(z_{1}, \mathbf{q}^{2}, \mu^{2}\right)\left[\tilde{P}_{g q}^{\prime}\left(\frac{z}{z_{1}}, \mathbf{p}, \mathbf{q}\right) \frac{z}{z_{1}}\right] \mathcal{Q}\left(\frac{x}{z},|\mathbf{q}+\mathbf{p}|^{2}\right)\right]
\end{aligned}
$$

where the gluonic part is given by momentum space formulation [36, 37] of the Balitsky-Kovchegov equation [38, 39]. In the above equation the parameter $R$ has an interpretation of radius of the hadron.

\section{Numerical studies}

First of all we would like to estimate the stability of eq. (3.26) with respect to the cutoff $\mu$. To address this issue we need to assume some form of quark distribution
$\mathcal{Q} 4$ To have a realistic form of this function we use the DLC 2016 set of parton densities 40.5 Employing this

${ }^{4}$ When a complete set of equations, including both quarks and gluons, will be available the quark distribution will be computed while solving this system.

5 The DLC 2016 PDFs are defined for $\mu>1.3 \mathrm{GeV}$. Since in our case $\mu$ is a technical cut-off which extends below this value, we 
set of PDFs (with just one quark flavor) we perform the convolution of the low $z$ and finite $z$ parts of the $P_{g q}$ kernel. We evaluate the quark density for $x=10^{-1}$ and $10^{-2}$ and we are lead to the conclusion that as $\mu^{2} \rightarrow 0$ the cutoff dependence gets weaker see Fig. 2. The other issue we can already address is the importance of the quark contribution to gluons at the unintegrated level as predicted by our equation. To answer this question we perform one iteration of the appropriate splitting functions on gluon and quark distribution in eq. (3.26), where for simplicity we again restrict to the case of one quark flavor. Since the applicability of the used gluon splitting function is limited to the low $x$ domain, we study the effects only for moderate $x$ values $x=\left\{10^{-1}, 10^{-2}\right\}$ of the longitudinal momentum fraction. From Fig. 3 we can see that at $x=10^{-2}$ the quark contribution is much smaller than the gluon contribution and can be neglected, while at larger $x$ it starts to be relevant.

\section{E. Low $x$ limit}

Since we are already working in the low- $x$ approximation for the gluon part it is natural to also study the result of Sec. IIIC when a similar limit is taken in case of $P_{g q}$ kernel. To do it in a correct way we need to go back to the kernel of eq. (2.4) and take the $z \rightarrow 0$ limit, which gives

$$
P_{g q}(z, \mathbf{p}, \mathbf{q})=C_{F} \frac{2}{z},
$$

and repeat the steps of Sec. III. If we do this and substitute the simplified kernel to eq. (3.13) it is easy to check that we will obtain

$$
\begin{aligned}
& \int_{c-i \infty}^{c+i \infty} \frac{d \omega}{2 \pi i} x^{-\omega} \frac{\alpha_{s}}{2 \pi} \frac{\omega}{\omega+\bar{\omega}} \int \frac{d^{2} \mathbf{p}}{\pi \mathbf{p}^{2}} \int_{0}^{1} d z z^{\omega} P_{g q}(z, \mathbf{p}, \mathbf{q}) \int_{0}^{1} d y y^{\omega-1} \mathcal{Q}\left(y,|\mathbf{q}+\mathbf{p}|^{2}\right) \theta\left(\mathbf{p}^{2}-\mu^{2}\right) \\
& =\int_{c-i \infty}^{c+i \infty} \frac{d \omega}{2 \pi i} x^{-\omega} \frac{\alpha_{s}}{\pi} C_{F} \frac{\omega}{\omega+\bar{\omega}} \int \frac{d^{2} \mathbf{p}}{\pi \mathbf{p}^{2}} \int_{0}^{1} d z z^{\omega-1} \int_{0}^{1} d y y^{\omega-1} \mathcal{Q}\left(y,|\mathbf{q}+\mathbf{p}|^{2}\right) \theta\left(\mathbf{p}^{2}-\mu^{2}\right) \\
& =\frac{\alpha_{s}}{\pi} C_{F} \int \frac{d^{2} \mathbf{p}}{\pi \mathbf{p}^{2}} \int_{x}^{1} \frac{d z}{z} z^{\bar{\omega}} \mathcal{Q}\left(\frac{x}{z},|\mathbf{q}+\mathbf{p}|^{2}\right) \theta\left(\mathbf{p}^{2}-\mu^{2}\right) .
\end{aligned}
$$

This leads to the final result (taking into account the nonlinear effects) in the low- $x$ limit

$$
\begin{aligned}
& \mathcal{F}\left(x, \mathbf{q}^{2}\right)=\tilde{\mathcal{F}}^{0}\left(x, \mathbf{q}^{2}\right) \\
& +\frac{\alpha_{s}}{\pi} \int \frac{d^{2} \mathbf{p}}{\pi \mathbf{p}^{2}} \theta\left(\mathbf{p}^{2}-\mu^{2}\right) \int_{x}^{1} \frac{d z}{z} \Delta_{R}\left(z, \mathbf{q}^{2}, \mu^{2}\right)\left[C_{A} \mathcal{F}\left(\frac{x}{z},|\mathbf{q}+\mathbf{p}|^{2}\right)+C_{F} \mathcal{Q}\left(\frac{x}{z},|\mathbf{q}+\mathbf{p}|^{2}\right)\right] \\
& -\frac{2 \alpha_{s}^{2}}{R^{2}} \int_{x}^{1} \frac{d z}{z} \Delta_{R}\left(z, \mathbf{q}^{2}, \mu^{2}\right)\left[\left(\int_{\mathbf{q}^{2}}^{\infty} \frac{\mathrm{d} l^{2}}{l^{2}} \mathcal{F}\left(\frac{x}{z}, l^{2}\right)\right)^{2}+\mathcal{F}\left(\frac{x}{z}, \mathbf{q}^{2}\right) \int_{\mathbf{q}^{2}}^{\infty} \frac{\mathrm{d} l^{2}}{l^{2}} \ln \left(\frac{l^{2}}{\mathbf{q}^{2}}\right) \mathcal{F}\left(\frac{x}{z}, l^{2}\right)\right] .
\end{aligned}
$$

We find that when the low- $x$ limit is taken, the treatment of the $\frac{1}{\mathbf{p}^{2}}$ singularity of the quark-to-gluon splitting appears to be in 1-1 correspondence with the low $x$ gluon-to-gluon splitting, i.e. the contributions of gluon $(\mathcal{F})$ and $(\mathcal{Q})$ in the second line of eq. 3.30 differ only by their overall color factor and are both regulated by a) the cut-off $\mu^{2}$ and b) the Regge form factor $\Delta_{R}$ which ensures stability in the limit $\mu \rightarrow 0$. Note that, since the treatment of the quark part of the evolution equation is

assume for this study that the quark distribution for $\mu<1.3$ $\mathrm{GeV}$ is constant. not affected by the presence of the non-linear terms in the second line of eq. 3.30 , we expect this equation to behave in the limit $\mu \rightarrow 0$ identical to its linear truncation. This is in particular true for the quark part which initially provided the main source of a potential instability; the purely gluonic non-linear evolution equation is on the other hand known to be stable in the limit $\mu \rightarrow 0$ [41.

\section{SUMMARY AND OUTLOOK}

In the paper we have constructed a nonlinear TMD evolution equation for gluons, receiving contribution 

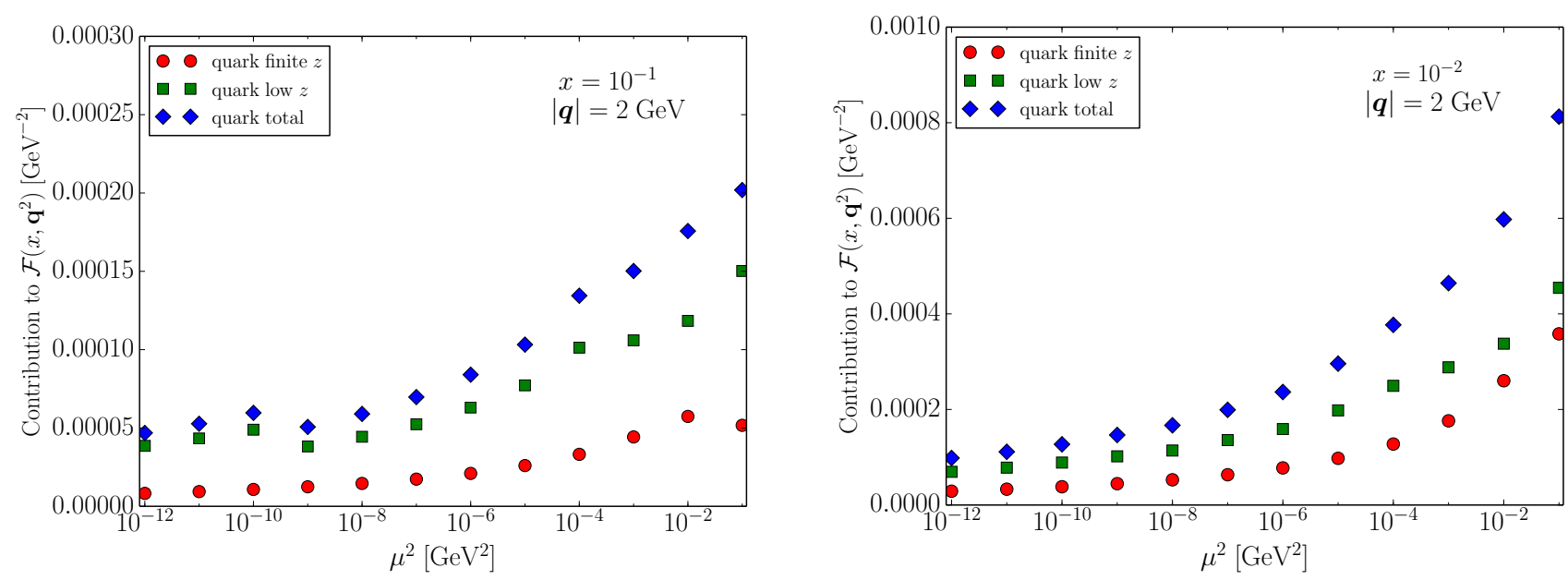

FIG. 2. The figure visualizes the cutoff dependence of the low $z$ and finite $z$ quark terms contributing to the gluon density.
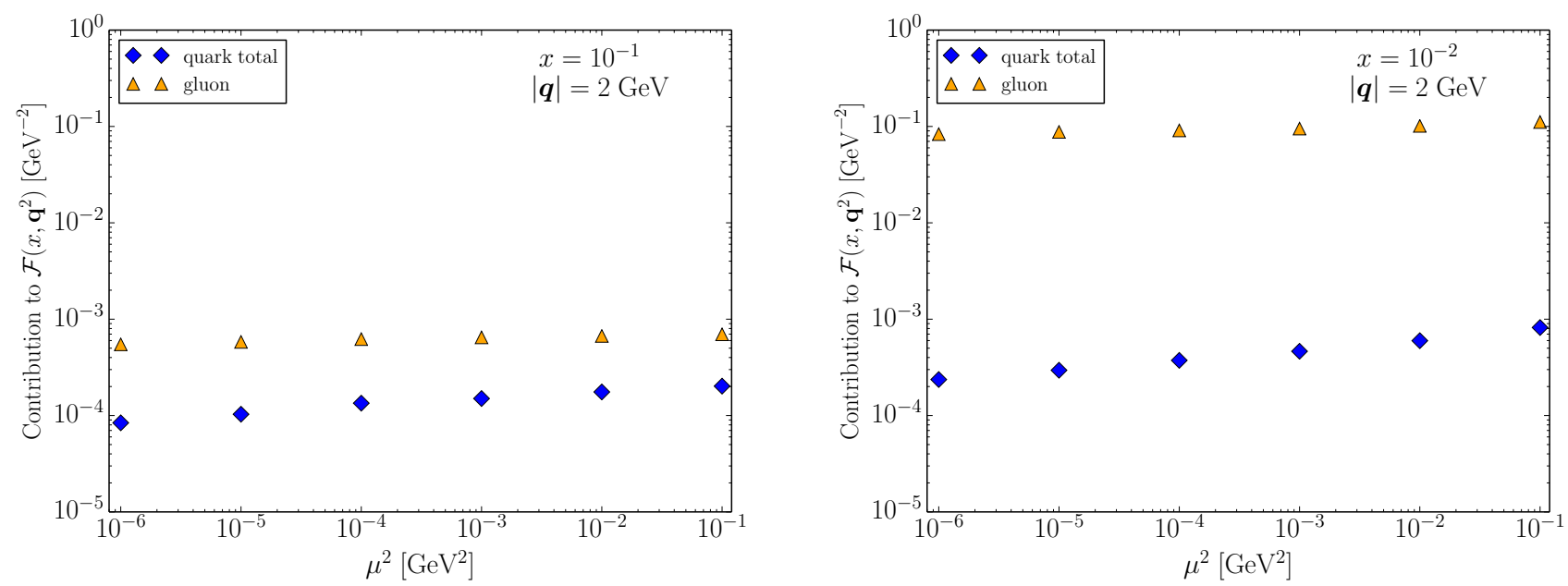

FIG. 3. The figure shows the relative contribution of gluon and quark terms at $|\mathbf{q}|=2 \mathrm{GeV}$ to the density of gluons for $x=10^{-1}$ (left) and $x=10^{-2}$ (right) as a function of cutoff $\mu$.

from quarks. In order to regularize the divergences of the $P_{g q}$ kernel, we introduced a cut-off and identified the latter with a similar cut-off introduced in the context of the BFKL kernel; within the BFKL kernel this cut-off serves for splitting off the low $p_{T}$ region of the real contributions and adding the latter to the virtual corrections. Resumming the combined contribution of 'virtual part and low $p_{T}$ real part' of the BFKL kernel to all orders in the strong coupling, one finds that both the pure gluonic contribution to the evolution equation as well as the quark induced term are finite if we send this cut-off to zero. In particular we demonstrated, via performing one iteration of the kernels, that the equation has a realistic chance to be stable against variation of the cutoff parameter, since after one iteration the result stabilizes. To perform a fully consistent study of the complete system of $k_{T}$-dependent evolution equations, we need to calculate the virtual contributions to the quark-to-quark splitting functions in the framework of $k_{T}$-factorization. We plan to address this question through a calculation of corresponding virtual corrections, using the calculational framework developed in [42,47] to determine loop-corrections within high-energy factorization. These results will then be combined with the already known real contributions, using an appropriate extension of the Curci Furmanski Petronzio method to off-shell initial quarks. We also expect that one can again perform a similar resummation of the combined virtual and low $p_{T}$ real terms, leading to a system of equations for quarks and gluons on equal footing. Furthermore, a numerical study of the complete system will then allow for a more consistent study of the cutoff dependence and - in addition - enable us to study the impact of nonlinearities on the quark density. 


\section{ACKNOWLEDGMENTS}

We acknowledge useful discussions with Hannes Jung and Francesco Hautmann at the early stage of the project. K.K has been supported by Narodowe Centrum Nauki with Sonata Bis grant DEC2013/10/E/ST2/00656. M.H. acknowledges support by CONACyT-Mexico grant numbers CB-2014-22117 and Proy. No. 241408.
[1] R. Angeles-Martinez et al., "Transverse Momentum Dependent (TMD) parton distribution functions: status and prospects," Acta Phys. Polon. B46 (2015), no. 12, 2501-2534, 1507.05267 .

[2] E. A. Kuraev, L. N. Lipatov, and V. S. Fadin, "Multi Reggeon Processes in the Yang-Mills Theory," Sov. Phys. JETP 44 (1976) 443-450. [Zh. Eksp. Teor. Fiz.71,840(1976)].

[3] I. I. Balitsky and L. N. Lipatov, "The Pomeranchuk Singularity in Quantum Chromodynamics," Sov. J. Nucl. Phys. 28 (1978) 822-829. [Yad. Fiz.28,1597(1978)].

[4] S. Catani, M. Ciafaloni, and F. Hautmann, "High-energy factorization and small $\mathrm{x}$ heavy flavor production," Nucl. Phys. B366 (1991) 135-188.

[5] S. Catani, M. Ciafaloni, and F. Hautmann, "GLUON CONTRIBUTIONS TO SMALL x HEAVY FLAVOR PRODUCTION," Phys. Lett. B242 (1990) 97-102.

[6] F. Dominguez, C. Marquet, B.-W. Xiao, and F. Yuan, "Universality of Unintegrated Gluon Distributions at small x," Phys. Rev. D83 (2011) 105005, 1101.0715

[7] P. Kotko, K. Kutak, C. Marquet, E. Petreska, S. Sapeta, and A. van Hameren, "Improved TMD factorization for forward dijet production in dilute-dense hadronic collisions," JHEP 09 (2015) 106, 1503.03421

[8] M. Deak, F. Hautmann, H. Jung, and K. Kutak, "Forward Jet Production at the Large Hadron Collider," JHEP 09 (2009) 121, 0908.0538.

[9] A. van Hameren, P. Kotko, and K. Kutak, "Helicity amplitudes for high-energy scattering," JHEP 01 (2013) 078, 1211.0961.

[10] A. van Hameren, P. Kotko, and K. Kutak, "Multi-gluon helicity amplitudes with one off-shell leg within high energy factorization," JHEP 12 (2012) 029, 1207.3332

[11] F. Hautmann, M. Hentschinski, and H. Jung, "Unintegrated sea quark at small $\mathrm{x}$ and vector boson production," in Proceedings, 47th Rencontres de Moriond on $Q C D$ and High Energy Interactions, pp. 391-394. 2012. 1209.6305

[12] F. Hautmann, H. Jung, and S. T. Monfared, "The CCFM uPDF evolution uPDFevolv Version 1.0.00," Eur. Phys. J. C74 (2014) 3082, 1407.5935.

[13] O. Gituliar, M. Hentschinski, and K. Kutak, "Transverse-momentum-dependent quark splitting functions in $k_{T}$-factorization: real contributions," JHEP 01 (2016) 181, 1511.08439

[14] S. Catani and F. Hautmann, "High-energy factorization and small $\mathrm{x}$ deep inelastic scattering beyond leading order," Nucl. Phys. B427 (1994) 475-524, hep-ph/9405388

[15] F. Hautmann, M. Hentschinski, and H. Jung, "Forward Z-boson production and the unintegrated sea quark density," Nucl. Phys. B865 (2012) 54-66, 1205.1759
[16] G. Curci, W. Furmanski, and R. Petronzio, "Evolution of Parton Densities Beyond Leading Order: The Nonsinglet Case," Nucl. Phys. B175 (1980) 27-92.

[17] S. Jadach, A. Kusina, M. Skrzypek, and M. Slawinska, "Two real parton contributions to non-singlet kernels for exclusive QCD DGLAP evolution," JHEP 08 (2011) $012,1102.5083$

[18] O. Gituliar, S. Jadach, A. Kusina, and M. Skrzypek, "On regularizing the infrared singularities in QCD NLO splitting functions with the new Principal Value prescription," Phys. Lett. B732 (2014) 218-222, 1401.5087

[19] S. Jadach, A. Kusina, W. Placzek, and M. Skrzypek, "On the dependence of QCD splitting functions on the choice of the evolution variable," JHEP 08 (2016) 092, 1606.01238 .

[20] M. Ciafaloni, "Coherence Effects in Initial Jets at Small q**2 / s," Nucl. Phys. B296 (1988) 49-74.

[21] S. Catani, F. Fiorani, and G. Marchesini, "Small x Behavior of Initial State Radiation in Perturbative QCD," Nucl. Phys. B336 (1990) 18-85.

[22] S. Catani, F. Fiorani, and G. Marchesini, "QCD Coherence in Initial State Radiation," Phys. Lett. B234 (1990) 339-345.

[23] G. Marchesini, "QCD coherence in the structure function and associated distributions at small x," Nucl. Phys. B445 (1995) 49-80, hep-ph/9412327

[24] M. A. Kimber, A. D. Martin, and M. G. Ryskin, "Unintegrated parton distributions and prompt photon hadroproduction," Eur. Phys. J. C12 (2000) 655-661, hep-ph/9911379.

[25] M. A. Kimber, A. D. Martin, and M. G. Ryskin, "Unintegrated parton distributions," Phys. Rev. D63 (2001) 114027, hep-ph/0101348.

[26] W. Schafer and A. Szczurek, "Low mass Drell-Yan production of lepton pairs at forward directions at the LHC: a hybrid approach," Phys. Rev. D93 (2016), no. $7,074014,1602.06740$

[27] R. Maciuła, A. Szczurek, and M. Łuszczak, "Open charm meson production at BNL RHIC within $k_{t}$-factorization approach and revision of their semileptonic decays," Phys. Rev. D92 (2015), no. 5, 054006, 1505.05038

[28] B. Ducloué, L. Szymanowski, and S. Wallon, "Evaluating the double parton scattering contribution to Mueller-Navelet jets production at the LHC," Phys. Rev. D92 (2015), no. 7, 076002, 1507.04735.

[29] M. A. Nefedov, V. A. Saleev, and A. V. Shipilova, "Dijet azimuthal decorrelations at the LHC in the parton Reggeization approach," Phys. Rev. D87 (2013), no. 9, 094030, 1304.3549 .

[30] S. P. Baranov, A. V. Lipatov, M. A. Malyshev, A. M. Snigirev, and N. P. Zotov, "Associated production of electroweak bosons and heavy mesons at $\mathrm{LHCb}$ and the 
prospects to observe double parton interactions," Phys. Rev. D93 (2016), no. 9, 094013, 1604.03025 .

[31] J. Kwiecinski, A. D. Martin, and P. J. Sutton, "Constraints on gluon evolution at small x," Z. Phys. C71 (1996) 585-594, hep-ph/9602320.

[32] K. Kutak, K. Golec-Biernat, S. Jadach, and M. Skrzypek, "Nonlinear equation for coherent gluon emission," JHEP 02 (2012) 117, 1111.6928

[33] K. Kutak, "Resummation in nonlinear equation for high energy factorisable gluon density and its extension to include coherence," JHEP 12 (2012) 033, 1206.5757

[34] L. V. Gribov, E. M. Levin, and M. G. Ryskin, "Semihard Processes in QCD," Phys. Rept. 100 (1983) $1-150$.

[35] F. Gelis, E. Iancu, J. Jalilian-Marian, and R. Venugopalan, "The Color Glass Condensate," Ann. Rev. Nucl. Part. Sci. 60 (2010) 463-489, 1002.0333

[36] K. Kutak and J. Kwiecinski, "Screening effects in the ultrahigh-energy neutrino interactions," Eur. Phys. J. C29 (2003) 521, hep-ph/0303209.

[37] J. Bartels and K. Kutak, "A Momentum Space Analysis of the Triple Pomeron Vertex in pQCD," Eur. Phys. J. C53 (2008) 533-548, 0710.3060

[38] Y. V. Kovchegov, "Small x F(2) structure function of a nucleus including multiple pomeron exchanges," Phys. Rev. D60 (1999) 034008, hep-ph/9901281

[39] I. Balitsky, "Operator expansion for high-energy scattering," Nucl. Phys. B463 (1996) 99-160, hep-ph/9509348.

[40] K. Kutak, R. Maciula, M. Serino, A. Szczurek, and A. van Hameren, "Four-jet production in single- and double-parton scattering within high-energy factorization," JHEP 04 (2016) 175, 1602.06814.

[41] K. Kutak, W. Płaczek, and D. Toton, "Numerical solution of the integral form of the resummed Balitsky-Kovchegov equation," Acta Phys. Polon. B44 (2013), no. 7, 1527-1535, 1303.0431

[42] M. Hentschinski and A. Sabio Vera, "NLO jet vertex from Lipatov's QCD effective action," Phys. Rev. $\mathbf{D 8 5}$ (2012) 056006, 1110.6741

[43] M. Hentschinski, "Pole prescription of higher order induced vertices in Lipatov's QCD effective action," Nucl. Phys. B859 (2012) 129-142, 1112.4509.

[44] G. Chachamis, M. Hentschinski, J. D. Madrigal Martinez, and A. Sabio Vera, "Quark contribution to the gluon Regge trajectory at NLO from the high energy effective action," Nucl. Phys. B861 (2012) 133-144, 1202.0649.

[45] G. Chachamis, M. Hentschinski, J. D. Madrigal Martinez, and A. Sabio Vera, "Forward jet production \& quantum corrections to the gluon Regge trajectory from Lipatov's high energy effective action," Phys. Part. Nucl. 45 (2014), no. 4, 788-799, 1211.2050

[46] G. Chachamis, M. Hentschinski, J. D. Madrigal Martinez, and A. Sabio Vera, "Next-to-leading order corrections to the gluon-induced forward jet vertex from the high energy effective action," Phys. Rev. D87 (2013), no. 7, 076009, 1212.4992

[47] G. Chachamis, M. Hentschinski, J. D. Madrigal Martinez, and A. Sabio Vera, "Gluon Regge trajectory at two loops from Lipatov's high energy effective action," Nucl. Phys. B876 (2013) 453-472, 1307.2591 